\title{
BLOOD PRESSURE MEASUREMENTS TAKEN BY PATIENTS ARE SIMILAR TO HOME AND AMBULATORY BLOOD PRESSURE MEASUREMENTS
}

\author{
Angela M. G. Pierina, Edna C. Ignez ${ }^{\text {a }}$, Wilson Jacob Filho ${ }^{\text {b }}$, Alfonso Júlio Guedes \\ Barbato $^{\mathrm{b}}$, Décio Mion Jr. ${ }^{\mathrm{b}}$
}

\begin{abstract}
Pierin AMG, Ignez EC, Jacob Filho W, Barbato AJG, Mion Jr. D. Blood pressure measurements taken by patients are similar to home and ambulatory blood pressure measurements. Clinics. 2008;63(1):43-50.
\end{abstract}

OBJECTIVE: To compare blood pressure measurements taken at home by physicians, nurses, and patients with office blood pressure measurement, ambulatory blood pressure monitoring and home blood pressure measurement.

METHODS: A total of 44 patients seen by a home care program were studied. Protocol 1 a) blood pressure was measured by the patient, a physician and a nurse during a regular home visit (Home1); b) home blood pressure measurement was measured for 4 days (HBPM1); c) office blood pressure measurement was measured by a physician, a nurse, and the patient; and by 24-hour ambulatory blood pressure monitoring. Protocol 2 blood pressure was measured by the patient, a physician, and a nurse during a special home visit in the presence of a physician and a nurse only (Home2); and b) home blood pressure measurement was taken for the second time (HBPM2). Echocardiography, guided by a two-dimensional echocardiograph, was performed.

RESULTS: Protocol 1: a) office blood pressure measurement and Home1 were significantly higher than ambulatory blood pressure monitoring, except for systolic and diastolic office blood pressure measurement taken by the patient or a family member, systolic blood pressure taken by a nurse, and diastolic blood pressure taken by a physician. b) ambulatory blood pressure monitoring and HBPM1 were similar. Protocol 2: a) HBPM2 and Home2 were similar. b) Home2 was significantly lower than Home1, except for diastolic blood pressure taken by a nurse or the patient. There were significant relationships between: a) diastolic blood pressure measured by the patient and the thickness of the interventricular septum, posterior wall, and left ventricular mass; and b) ambulatory and HBPM2 diastolic and systolic blood pressure taken by a physician (home2) and left ventricular mass. Therefore, the data indicate that home blood pressure measurement and ambulatory blood pressure monitoring had good prognostic values relative to "office measurement."

CONCLUSION: This study showed that the measurement most similar to home blood pressure measurement and ambulatory blood pressure monitoring was blood pressure measured by the patient, and that home blood pressure measurement and ambulatory blood pressure monitoring had good prognostic value relative to "office measurements".

KEYWORDS: Home blood pressure. Ambulatory blood pressure. Office blood pressure measurement. Hypertension. White coat effect.

\section{INTRODUCTION}

Office BP has important limitations. It is usually higher than readings taken at home or by ambulatory monitoring. The office environment and the presence of a physician can cause a rise in blood pressure, leading to a situation described as the white coat effect, which is so named because

\footnotetext{
a School of Nursing, Universidade de São Paulo - São Paulo/SP, Brazil.

${ }^{\text {b }}$ Faculdade de Medicina da Universidade de São Paulo - São Paulo/SP, Brazil. pierin@usp.br

Received for publication on September 04, 2007.

Accepted for publication on October 22, 2007.
}

of its association with physicians' clothes. The white coat effect is usually defined by an increase in blood pressure measured in the office over the daytime mean obtained by ambulatory blood pressure monitoring (ABPM). The white coat effect may be observed in normotensive people as well. However, it should be differentiated from white coat hypertension which is characterized by a persistent hypertension in the office in combination with a normal ambulatory or home BP. ${ }^{1,2}$ Therefore, ABPM and HBPM may be useful when evaluating patients for the white coat effect. Additionally, prospective studies have shown that ABPM correlates with target organ damage better than office blood 
pressure measurements ${ }^{3,4}$ and, more recently, this correlation has been shown for HBPM was well.,

The presence of a physician is not the only factor that can lead to a rise in blood pressure. A pioneering study carried out in this area approximately two decades ago showed that the presence of a physician or a nurse led to a rise in blood pressure. However, the rise caused by the presence of a nurse was lower than that caused by a physician. The authors concluded that repeated physician's visits within a short period of time did not prevent a rise in blood pressure, but a decrease in blood pressure could be observed if the measurement was taken by a nurse. ${ }^{7}$ A more recent study demonstrated that blood pressure measurements taken by a nurse are significantly lower than those obtained by a physician. ${ }^{8}$

The presence of an observer, a physician or a nurse, and the office environment increase blood pressure. Therefore, who would be the best person and where would be the best place to measure blood pressure? A study revealed that blood pressure self-measurement in the office did not decrease the white coat effect. ${ }^{9}$ The other question is if the white coat effect could be avoided or decreased if BP was measured at the patient's home by a physician or a nurse.

Therefore, the main objective of this study was to compare BP measurements at the patient's home performed by a physician, a nurse, or the patient/family member with BP measurement obtained either in the office or by ABPM and HBPM. The secondary objectives were to correlate BP measurements with echocardiographic patterns and to assess the white coat effect.

\section{METHODS}

This study was carried out at the Home Health Care Center of a University General Hospital. This center aims at providing full care to patients with chronic diseases and to those who need out-of-hospital care. The most common professional home care provides regular home visits, including physician care, nursing, physical therapy, medical social work and nutritional and psychological care. Therefore, this service sees a great number of hypertensive people who need blood pressure assessment so that hypertension can be diagnosed and antihypertensive therapy can be assessed. This study was approved by the Ethics Committee of the hospital to comply with Decree 196/96 by the National Health Council and Declarations of Human Rights of Helsinki.

Forty-four patients were studied (49\% women, $77 \%$ white, $68 \pm 15$ years and $82 \%$ hypertensive treated with antihypertensive medications). Patients were included in the study after giving written informed consent. All patients, or a family member when the patient was unable, were instructed individually in the use of an electronic blood pressure measuring device.

The study was divided into two phases - protocol 1 and protocol 2 (Figure 1).

\section{Protocol 1}

During a regular home visit (Home1) in the presence of several health professionals, a blood pressure measurement was taken. In this case, blood pressure was measured by the patient or a family member, by a physician, and by a nurse. After that, home blood pressure measurements were taken on four days during the following week (HBPM1). The patient then went to the hospital for a regular appointment after two weeks. In the office, blood pressure was measured by a physician, by a nurse, and by the patient or a family member. After that, 24-hour ambulatory blood pressure monitoring was performed (ABPM).

\section{REGULAR HOME VISIT}

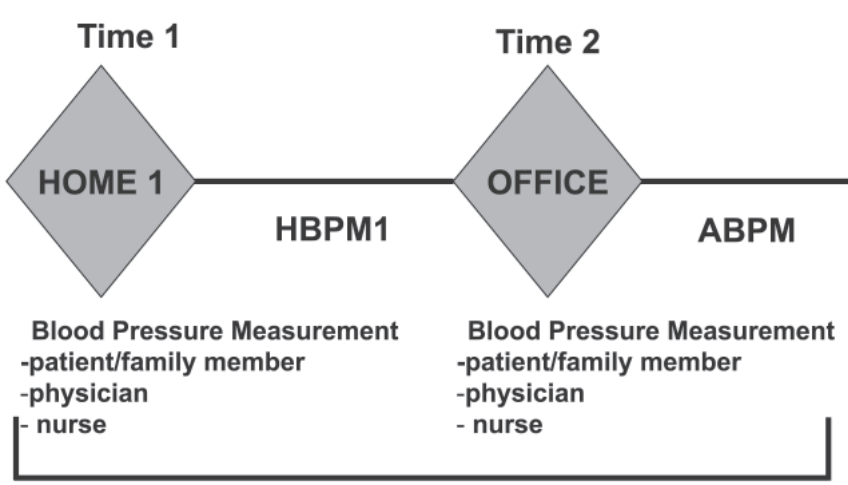

\section{Protocol 1}

SPECIAL HOME VISIT

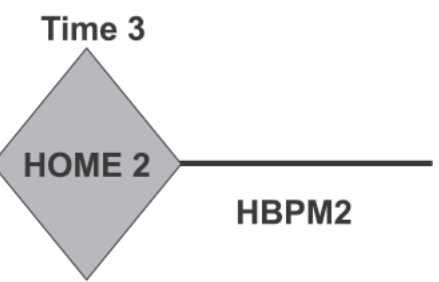

Blood Pressure Measurement -patient/family member -physician - nurse

Figure 1 - Blood Pressure Measurement 


\section{Protocol 2}

During a special home visit (Home2) to the same patients as in Protocol 1 in the presence of the physician and nurse, a blood pressure measurement was taken. In this case, blood pressure was measured by the patient or a family member, by a physician, and by a nurse. After that, a home blood pressure measurement was taken a second time during the following week (HBPM2).

Blood Pressure Measurements taken by a physician, a nurse and the patient/family member

Blood pressure measurements taken by a physician, a nurse and the patient or a family member followed the same procedure regardless of the location (home or office). An automatic device (OMRON - HEM 705CP, Omron Corporation Tokyo Japan) and an appropriate cuff size for the patient's left arm circumference were used. The patient was in a sitting position, with the arm at heart level and the forearm resting on a table. Blood pressure was measured after a 5 to 10 -minute rest 3 times with a 1 to 2 -minute interval between the measurements. The mean of these three measurements was considered for analyses.

The sequence of blood pressure measurements taken by the patient or family member, the physician, and the nurse was established by a randomization table.

\section{Home Blood Pressure Measurement}

Home blood pressure measurement was obtained by using an appropriate cuff size for the left arm circumference with the patient in a sitting position; after a 5-minute rest, measurements were taken twice with a 1 to 2-minute interval between the measurements. Sets of measurements were taken at three different times of day: morning (07.0008.00 a.m.), afternoon (02.00-03.00 p.m.) and night (07.0008.00 p.m.) on four consecutive days.

\section{Ambulatory Blood Pressure Monitoring}

Twenty-four hour ambulatory blood pressure monitoring was performed using an oscillometric device (Spacelabs 90207, Spacelabs Inc, Redmond, Washington, USA). Measurements were taken every 15 minutes during the daytime (from 07.00a.m. to 10.00 p.m.) and every 20 minutes at nighttime (from 10.00p.m. to 07.00 a.m.) in the nondominant arm. An appropriate cuff size was used for the patient's arm circumference.

\section{Echocardiography}

M-mode echocardiography, guided by two-dimensional echocardiography with the patient maintained in a partial left decubitus position, was performed using a General
Electric Vivid 7 Instrument (Milwaukee, Wisconsin, USA). M-mode measurements were taken according to the American Society of Echocardiography recommendations. ${ }^{10}$ Left ventricular mass was determined using the autopsy-validated Devereux's formula. ${ }^{11}$ All echocardiograms were analyzed by the same physician, who did not know the patients' clinical conditions.

\section{Statistical analysis}

Continuous variables are given as mean \pm standard deviation. For data analysis, blood pressure measurements taken by a physician, a nurse, and the patient or a family member at the office and at home were compared to each other and to ABPM and HBPM using repeated analysis of variance. To test relations between the echocardiographic patterns and blood pressure measurements, stepwise regression analysis and Pearson's correlation coefficient were performed, and $\mathrm{p}<0.05$ was considered statistically significant.

\section{RESULTS}

\section{Protocol 1}

Comparison of blood pressure measurements taken by the patient/family member, physician and nurse during a regular home visit (Home 1) and in the office with ABPM and HBPMI.

The averages of systolic and diastolic BP obtained by ABPM $(124.2 \pm 12.6 / 74.6 \pm 10.4 \mathrm{~mm} \mathrm{Hg})$ and by HBPM1 $(123.6 \pm 19 / 74.1 \pm 10.0 \mathrm{~mm} \mathrm{Hg}$ ) were similar (Figure 2). Systolic and diastolic blood pressure measurements taken by a physician in the office $(132.2 \pm 20.3 / 77.4 \pm 12.9 \mathrm{~mm} \mathrm{Hg})$ and in Homel during a regular home visit (135.6 $\pm 21.3 /$ $79.8 \pm 12.2 \mathrm{~mm} \mathrm{Hg}$ ) were similar; however, the measurements were significantly higher $(\mathrm{p}<0.05)$ than those obtained by ABPM and HBPM1, except for the office diastolic blood pressure, which was similar to ABPM and HBPM1.

Diastolic blood pressure measurements taken by a nurse in the office $(128.1 \pm 21.9 / 76.7 \pm 13.3 \mathrm{~mm} \mathrm{Hg})$ and at Home 1 $(134.9 \pm 22.0 / 78.8 \pm 13.2 \mathrm{~mm} \mathrm{Hg})$ and those obtained by ABPM and HBPM1 were similar. Systolic blood pressure taken by a nurse was observed to be: a) lower in the office than during the home visit and similar to that obtained by ABPM and HBPM1; and b) higher during regular home visits than measured by ABPM and HBPM1.

The blood pressure measurements taken by the patient or a family member showed that: a) they were similar in the office and during regular home visits (Home1); b) office blood pressure measurements were similar to those obtained by ABPM and HBPM1; and c) blood pressure 


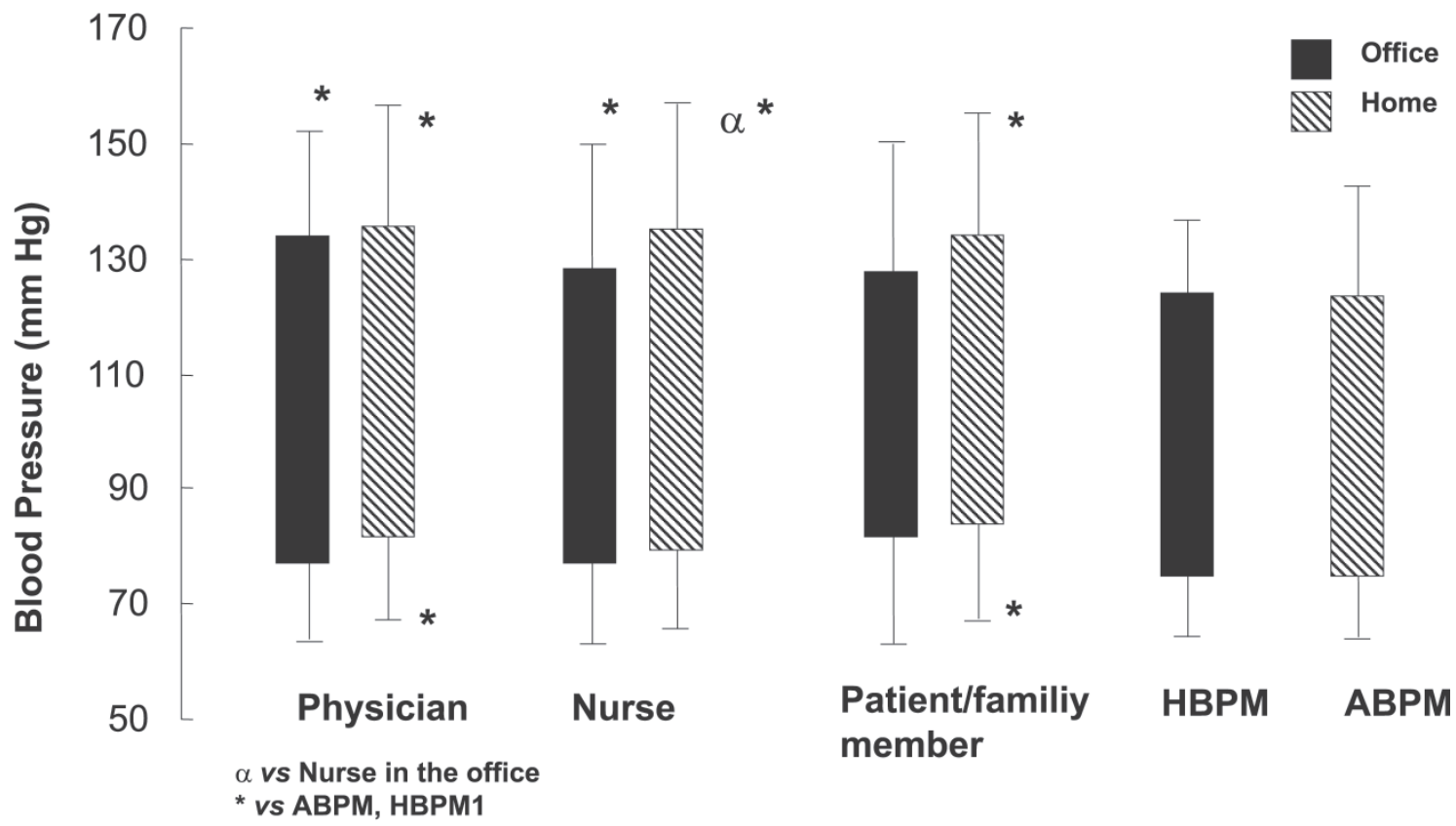

Figure 2 - Blood pressure measurement in office and during a regular home visit (Home 1) taken by patients/family member, physicians, and nurses compared with Ambulatory Blood Pressure measurement (ABPM) and Home Blood Pressure Measurement (HBPM1).

measurements taken at Home1 were significantly higher $(\mathrm{p}<0.05)$ than those obtained by ABPM and HBPM1.

\section{Protocol 2}

Blood pressure measured at home by the patient/family member, physician, and nurse during a special home visit (Home2) compared with the second home blood pressure measurement (HBPM2).

There was no significant difference between blood pressure measurements taken by a physician, a nurse and the patient or a family and HBPM2 performed during a special home visit (Home 2) in the presence of a physician and nurse (Table 1).

Table 1 - Blood pressure measurements (mean \pm standard deviation, $\mathrm{mm} \mathrm{Hg}$ ) taken by a physician, a nurse and the patient/family member during a special home visit (Home 2) and HBPM2.

\begin{tabular}{lcc}
\hline & \multicolumn{2}{c}{ Blood Pressure } \\
& Systolic & Diastolic \\
\hline HBPM2 & $123 \pm 21$ & $74 \pm 10$ \\
Physician- home2 & $126 \pm 23$ & $75 \pm 11$ \\
Nurse- home2 & $125 \pm 21$ & $75 \pm 12$ \\
Patient/family member- home2 & $124 \pm 24$ & $76 \pm 13$ \\
\hline
\end{tabular}

HBPM2: home blood pressure measurement taken after Home2
Correlation between blood pressure measurements taken by a physician, a nurse and the patient/family member at home (Home 2), HBPM2 and ABPM with echocardiographic parameters (Table 2).

The univariate analysis showed that systolic and diastolic blood pressure measurements taken by a nurse and systolic BP measurements taken by a physician were correlated $(\mathrm{p}<0.05)$ with the thickness of the interventricular septum and the posterior wall of the left ventricle. Diastolic blood pressure measurements taken by the patient or a family member correlated with interventricular septum thickness, posterior wall thickness and left ventricular mass.

Multivariate analysis of the variables revealed a significant relationship $(\mathrm{p}<0.05)$ between diastolic BP measurements taken by the patient/family member and the three echocardiographic parameters assessed: interventricular septum thickness, posterior wall thickness and left ventricular mass. Diastolic blood pressure measurements obtained by ABPM and HBPM and systolic BP measurements taken by a physician correlated with left ventricular mass.

\section{White Coat Effect}

The white coat effect was assessed by taking into account differences between measurements taken in the office by a physician, a nurse, and the patient/family member and HBPM1. Considering positive differences for systolic and diastolic BP in relation to measurements taken 
Table 2 - Multivariate analysis and correlation ( $\mathrm{r}$ value / $\mathrm{p}$ value) between posterior wall dimensions, interventricular septum and left ventricular mass (LVM) and Home2, HBPM2 and ABPM.

\begin{tabular}{lcccccc}
\hline & \multicolumn{2}{c}{ Interventricularseptum } & \multicolumn{2}{c}{ Posterior Wall } & \multicolumn{2}{c}{ LVM } \\
Blood Pressure & $\mathrm{r} / \mathrm{p}$ & Multiv. Analysis & $\mathrm{r} / \mathrm{p}$ & Multiv. Analysis & $\mathrm{r} / \mathrm{p}$ & $\mathrm{Multiv}$. Analysis \\
\hline Systolic ABPM & $0.21 / 0.47$ & $\mathrm{~ns}$ & $0.22 / 0.45$ & $\mathrm{~ns}$ & $-0.13 / 0.66$ & $\mathrm{~ns}$ \\
Diastolic ABPM & $0.09 / 0.75$ & $\mathrm{~ns}$ & $0.11 / 0.71$ & $\mathrm{~ns}$ & $-0.27 / 0.35$ & $\mathrm{p}<0.05$ \\
Systolic HBPM2 & $-0.04 / 0.87$ & $\mathrm{~ns}$ & $-0.03 / 0.09$ & $\mathrm{~ns}$ & $-0.34 / 0.18$ & $\mathrm{~ns}$ \\
Diastolic HBPM2 & $0.22 / 0.40$ & $\mathrm{~ns}$ & $0.16 / 0.53$ & $\mathrm{~ns}$ & $-0.21 / 0.42$ & $\mathrm{p}<0.05$ \\
Physician2-Systolic BP & $0.49 / 0.04$ & $\mathrm{~ns}$ & $0.48 / 0.048$ & $\mathrm{~ns}$ & $0.19 / 0.46$ & $\mathrm{p}<0.05$ \\
Physician2 -Diastolic BP & $0.21 / 0.42$ & $\mathrm{~ns}$ & $0.23 / 0.37$ & $\mathrm{~ns}$ & $0.22 / 0.39$ & $\mathrm{~ns}$ \\
Nurse2 -systolic BP & $0.52 / 0.03$ & $\mathrm{~ns}$ & $0.52 / 0.03$ & $\mathrm{~ns}$ & $0.30 / 0.24$ & $\mathrm{~ns}$ \\
Nurse2 - diastolic BP & $0.55 / 0.02$ & $\mathrm{~ns}$ & $0.52 / 0.03$ & $\mathrm{~ns}$ & $0.42 / 0.09$ & $\mathrm{~ns}$ \\
Patient 2 -Systolic BP & $0.49 / 0.09$ & $\mathrm{~ns}$ & $0.38 / 0.13$ & $\mathrm{~ns}$ & $0.13 / 0.60$ & $\mathrm{~ns}$ \\
Patient2 - diastolic BP & $0.61 / 0.01$ & $\mathrm{p}<0.05$ & $0.64 / 0.005$ & $\mathrm{p}<0.05$ & $0.53 / 0.03$ & $\mathrm{p}<0.05$ \\
\hline
\end{tabular}

Home2: blood pressure measured by a physician, a nurse and the patient/family member during a special home visit in the presence of the physician and nurse; HBPM2: home blood pressure measurement taken after Home2; ABPM: ambulatory blood pressure monitoring; ns:not significant.

by the physician, it was observed that $35 \%$ of the patients experienced a white coat effect. This percentage decreased by $27 \%$ when measurements were taken by a nurse, and by $22 \%$ when they were taken by the patient/family member. The data observed in Table 3 shows that the magnitude of the white coat effect was around the level of 10 $\mathrm{mm} \mathrm{Hg}$, though differences over $30 \mathrm{~mm} \mathrm{Hg}$ were found.

Table 3 - Percent differences between office measurements taken by a physician, a nurse and the patient/family member and home blood pressure measurement (HBPM1).

Differences between office measurement and HBPM1 (mm Hg)

\begin{tabular}{lcccc}
\hline & 1 to 10 & 11 to 20 & 21 to 30 & $>30$ \\
\hline Physician-systolic BP (\%) & 22 & 7 & 10 & 7 \\
Physician-diastolic BP (\%) & 25 & 20 & - & - \\
Nurse - systolic BP (\%) & 22 & 5 & 10 & 5 \\
Nurse - diastolic BP (\%) & 30 & 13 & - & 3 \\
Patient-systolic BP (\%) & 30 & 13 & - & 3 \\
Patient-diastolic BP (\%) & 32 & 10 & - & 3 \\
\hline
\end{tabular}

HBPM1: home blood pressure measurement taken after Home1

There was no difference in white coat effect when considering the differences between the measurements taken by a physician, a nurse and the patient/family member and HBPM1. However, when assessed by ABPM, the white coat effect regarding systolic BP measured by a physician was significantly higher $(\mathrm{p}<0.05)$ than the white coat effect caused by the systolic BP measurement taken by a nurse (Table 4).

\section{DISCUSSION}

The main outcomes of the present study showed that blood pressure measurements taken in the presence of all
Table 4 - White Coat Effect (mean \pm standard deviation) differences between measurements taken by a physician, a nurse and the patient/ family member in the office, and ambulatory (ABPM) and home (HBPM1) blood pressures.

\begin{tabular}{lll}
\hline & HBPM1 & ABPM \\
\hline Physician-systolic BP & $3.4 \pm 20.4$ & $8.8 \pm 14.9 *$ \\
Physician-diastolic BP & $1.8 \pm 9.6$ & $5.1 \pm 9.9$ \\
Nurse - systolic BP & $1.34 \pm 20.5$ & $5.0 \pm 14.3$ \\
Nurse - diastolic BP & $1.1 \pm 10.3$ & $3.3 \pm 9.2$ \\
Patient - systolic BP & $1.8 \pm 21.3$ & $5.9 \pm 15.5$ \\
Patient - diastolic BP & $1.6 \pm 10.3$ & $4.8 \pm 10.4$ \\
\hline
\end{tabular}

* $\mathrm{p}<0.05$, physician - systolic BP vs. nurse - systolic BP

HBPM1: home blood pressure measurement taken after Home1

ABPM: ambulatory blood pressure monitoring

home health care professionals during a regular home visit were higher than those taken during a special home visit in the presence of the physician and nurse only. Such an outcome can indicate that the presence of an observer during the second blood pressure measurement during a special home visit did not influence the blood pressure measurement. It is also important to point out that blood pressure measured in the hospital by the patient/family member was similar to that found by ABPM and HBPM. Therefore, these data show that the collective home visit reproduced the hospital environment, leading to a rise in blood pressure. Blood pressure measurements taken by the patient/family member in a calm environment with the patient alone in a room most closely approximated the measurements obtained by ABPM and HBPM.

This study also confirmed findings reported in the literature. Systolic blood pressure measurements obtained in a hospital and in a doctor's office are similar to each other, and both are similar to values obtained by ABPM and HBPM. ${ }^{12,13,14}$ A recent investigation that monitored blood pressure in pharmacies showed a similar outcome. ${ }^{15}$ The dif- 
ferences found between measurements in and out of the hospital environment characterized the white coat effect. In the present study, the white coat effect, assessed by the differences between HBPM and the measurements taken by the patient/family member, physician and nurse, was not different. On the other hand, when the white coat effect was assessed by ABPM, the effect was significantly higher $(\mathrm{p}<0.05)$ with regard to systolic BP measurement taken by a physician than the white coat effect caused by a nurse. It was also observed that a greater percentage of patients (35\%) showed a white coat effect caused by measurements taken by a physician when compared to those taken by a nurse $(27 \%)$ and by the patient/family member (22\%). The presence of a physician as a factor that can cause an alarming reaction and a rise in blood pressure has been studied often. Mancia et al., in the 1980s, showed blood pressure variations caused by the presence of a physician. ${ }^{16}$ In a more recent trial, higher blood pressure levels were also observed more often when blood pressure was measured by a physician compared to measurements obtained by a nurse. ${ }^{8}$

The reasons for the white coat effect and white coat hypertension are not well known. Pickering believes that white coat hypertension can be caused by a stress response. ${ }^{17}$ However, its persistence in subsequent visits could not be explained by this mechanism. Blood pressure measurement is not simply a mechanical act; it has many facets, including the presence of a physician, the office environment and the reactions caused by the technique used for blood pressure measurement. These factors are likely associated with fear and anxiety, provoking stimuli with a feedback conditioned to a rise in blood pressure. However, studies showed that personality traits and tests of mental and physical stress reactivity did not determine the occurrence of a white coat effect. ${ }^{18,19}$

Another finding of note was that blood pressure measurements taken by the patient/family member in the hospital were similar to those obtained by ABPM and HBPM. Such measurements were even lower than those obtained by the patient/family member during regular home visits. Conditions such as a calm environment and the patient being alone or with a family member in the room and not in the presence of strangers created a situation in which the environment and an observer - a physician or nurse - could not interfere with the measurement; BP measurement by a family member, even in a clinical/hospital setting, did not cause the white coat effect. This observation could be explained by the patient's familiarity with this person and their routine together. Stergiou et al verified similar outcomes when they observed that self-measurement was not different from that taken by a family member. ${ }^{20}$

Casual blood pressure measurement taken by a physi- cian in the office has been the standard method most often applied to diagnose and treat hypertension. However, recent investigations have shown that casual measurement in the office is not the best method for blood pressure assessment due to blood pressure variability and errors related to the observer, the equipment, and the environment. After ABPM was created in the 1960s, several of the factors that interfere with blood pressure measurement could be controlled. ${ }^{21}$ Studies ${ }^{22,23}$ have revealed that ABPM shows better correlation with target-organ damage than measurements in the office and has a higher predictive value for cardiovascular morbidity and mortality. ${ }^{24,25}$ Home blood pressure measurement has also been suggested as a method for blood pressure assessment that can decrease or avoid errors related to office measurements. Like ABPM, home blood pressure measurement can also be useful for assessment of the white coat effect and antihypertensive treatment management. It is better correlated with target-organ damage than office measurement. ${ }^{26,27,28,29}$

The different methods for blood pressure measurement in the present study were also assessed for a correlation with echocardiographic parameters. In the multivariate analysis, it was observed that the correlation level (r) presented increasing values for the septum and the posterior wall ( 0.49 and 0.48 , respectively) when systolic BP measurements was taken by a physician, when systolic $(0.52$ and 0.52$)$ and diastolic (0.55 and 0.52) BP measurements were taken by a nurse and when diastolic BP measurements were taken by the patient/family member for the three parameters assessed: interventricular septum (0.61), the thickness of posterior wall (0.64), and the mass of left ventricle (0.53). However, diastolic BP obtained by ABPM and systolic BP obtained by HBPM only correlated with the echocardiographic findings of left ventricular mass and posterior wall, respectively. The small associations observed for ABPM and HBPM are one of the limitations of the study which may be explained by the small number of patients (22) who were in a good enough condition to be assessed by echocardiograms, i.e., not affected by old age or pre-existing cardiac alterations.

\section{CONCLUSION}

Blood pressure measurements taken by a physician, a nurse, and the patient/family member during a regular home visit at the patient's house in the presence of all home care professionals were similar to those obtained in the office, and when home blood pressure measurements were taken during special home visits in the presence of a physician and a nurse only, blood pressure values were lower and more similar to those obtained by HBPM; therefore, they were not influenced by the presence of an observer. The 
outcomes suggest that blood pressure assessment of patients who are seen by home care professionals is also influenced by the factors that interfere with office blood pressure measurement. Another important point is that the best person to measure blood pressure in the office would be the pa- tient or a family member alone in the room using an automatic device. Another interesting finding was that HBPM was similar to ABPM and, as it is cheaper, can be useful for both the management of hypertension and for predicting prognostic indications.

\section{REFERENCES}

1. Pickering TG, Gerin W, Schwartz AR. What is the white coat effect and how should it be measured? Blood Press Monit. 2002;7:293-300.

2. Verdecchia P, Schillaci G, Borgioni C, Ciucci A, Zampi I, Gattobigio R.et al. White coat hypertension and white coat effect. Similarities and differences. Am J Hypertens. 1995;8:790-8.

3. Imai Y. Prognostic significance of ambulatory blood pressure. Blood Press Monit. 1999;4:249-59.

4. Staessen JA, Thijs L, Fagard R, O'Brien ET, Clement D, de Leeuw PW et al. Predicting cardiovascular risk using conventional vs ambulatory blood pressure in older patients with systolic hypertension. JAMA. $1999 ; 282: 539-46$

5. Tsunoda S, Kawano Y, Horio T, Okuda N, Takishita S. Relationship between home blood pressure and longitudinal changes in target organ damage in treated hypertensive patients. Hypertens Res. 2002;25:16773.

6. Asayama K, Ohkubo T, Kikuya M, Metoki H, Hoshi H, Hashimoto J, et al. Prediction of stroke by self-measurement of blood pressure at home versus casual screening blood pressure measurement in relation to the Joint National Committee7 Classification The Ohasama Study. Stroke. 2004;35:2356-61.

7. Mancia G, Parati G, Pomidossi G, Grassi G, Casadei R, Zanchetti A.. Alerting reaction and rise in blood pressure during measurement by physician and nurse. Hypertension. 1987;9:209-15.

8. La Batide-Alanore A, Chatellier G, Bobrie G, Fofol I, Plouin PF Comparison of nurse and physician determined clinic blood pressure levels in patients referred toa a hypertension clinic:complications for subsequent management. J Hypertens. 2000;18:391-8.

9. Myers MG, Meglis G, Polemidiotis G. The impact of physician vs automated blood pressure reading on office-induced hypertension. J Hum Hypertens. 1997;11:491-3.

10. Sahan DJ, de maria A, Weisman A. The Committee on M - Mode Standardization of the American Society of Echocardiography. Circulation. 1978;58:1072-3.

11. Devereux RB, Reichnek N. Echocardiographic determination of left ventricular mass in man. Anatomic validation of the method. Circulation. $1077 ; 55: 613-8$
12. Staessen JA, Byttebier G, Buntinx F, Celis H, O’Brien ET, Fagard R. Ambulatory blood pressure monitoring and treatment of hypertension investigators. Antihypertensive treatment based on conventional or ambulatory blood pressure measurement a randomized controlled trial. JAMA. 1997;278:1065-72.

13. Omvik P, Gehardsen G. The Norwegian office-, home-, and ambulatory blood pressure study (NOHA). Blood Pressure. 2003;12:211-9.

14. Little P, Barnett J, Barnsley L, Marjoram J, Fitzgerald-Barron A, Mant D.. Comparison of agreement between different measures of blood pressure in primary care and daytime ambulatory blood pressure. BMJ. 2002;325:254-8.

15. Botomino A, Martina B, Ruf D, Bruppacher R, Hersberger KE. White coat effect hypertension in community pharmacy practice. Blood Press Monit. 2005;10:13-8.

16. Mancia G, Bertinieri G, Grassi G, Parati G, Pomidossi G, Ferrari A al. Effects of blood pressure measurement by the doctor on patient's blood pressure and heart rate. Lancet. 1983;4:695-8.

17. Pickering TG. Ambulatory monitoring and blood pressure variability. London, Science Press, 1991.

18. Siegel WC, Blumenthal JA, Divine, GW. Physiological, psychological and behavioral factors and white coat hypertension. Hypertension. 1990;16:140-6.

19. Cardillo F, Falcao RP, Rossi MA, Mengel J. Psychophysiological reactivity and cardiac end-organ changes in white coat hypertension. Hypertension. 1993;21:836-44.

20. Stergiou GS, Efstathiou SP, Alamara CV, Mastorantonakis SE, Roussias LG. Home or self blood pressure measurement? What is the correct term? J Hypertens. 2003;21:2259-64.

21. Sokolow M, Werdegar D, Kain H, Hinman A. Relationship between level of blood pressuremeasured casually and by portable recorders and severity of complications in essential hypertension. Circulation. 1966;34:279-98.

22. Zanchetti A, Bond MG, Hennig M, Neiss A, Mancia G, Dal Palu C. et al. Risk factors associated with alterations in carotid intima-media thickness in hypertension: baseline data from the European Lacidipine Study on Atherosclerosis. J Hypertens. 1998;16:949-61. 
23. Shimada K, Kawamoto A, Matsubayashi K, Ozawa T. Silent cerebrovascular disease in the elderly: correlation with ambulatory pressure. Hypertension. 1990;16:692-9.

24. Mancia G, Zanchetti A, Agabiti-Rosei E, Benemio G, De Cesaris R, Fogari R et al. For the SAMPLE study Group: Ambulatory blood pressure is superior to clinic blood pressure in predicting treatmentinduced regression of left ventricular hypertrophy. Circulation. 1997:95:1464-70.

25. Ohkubo T, Imai Y, Tsuji I, Nagai K, Watanabe $\mathrm{N}$, Minami $\mathrm{N}$ et al. Prediction of mortality by ambulatory blood pressure monitoring versus screening blood pressure measurements:a pilot study on Ohasama. J Hypertens. 1997;15:357-64.

26. Mule G, Caimi G, Cottone S, Nardi E, Andronico G, Piazza G et al. Value of home blood pressure as predictor to target organ damage in mild arterial hypertension. J Cardiovasc Risk. 2002;9:123-9.
27. Asayama K, Ohkubo T, Kikuya M, Metoki H, Hoshi H, Hashimoto J et al. Prediction of Stroke by self-measurement of blood pressure at home versus casual screening blood pressure measurement in relation to the Joint national Committee 7 Classification The Ohasama Study. Stroke. 2004;35:2346-61

28. Imai Y, Ohkubo T, Kikuia M, Hashimoto J. Practical aspect of monitoring hypertension based on self measured blood pressure at home. Intern Med. 2004;43:771-8.

29. Ohkubo T, Obara T, Funahashi J, Kikuya M, Asayama K, Metoki H et al. Control of blood pressure as measured at home and office and comparison with physicians' assessment of control among treated hypertensive patients in Japan:First Report of the Japan Home versus Office Blood Pressure Measurement Evaluation (J-Home) Study. Hypertens Res. 2004;27:755-63. 